\title{
Quantum effects in electrical transport properties of Bismuth chalcogenides Topological Insulators
}

\author{
José A. Paixão ${ }^{1, *}$, Marta S.C. Henriques ${ }^{1}$, Carlotta Micale $^{2}$, Elsa B. Lopes ${ }^{2}$, Vanda M. \\ Pereira $^{3}$, and António P. Gonçalves ${ }^{2}$ \\ ${ }^{1}$ CFisUC, Department of Physics, University of Coimbra, P-3004-516 Coimbra, Portugal \\ ${ }^{2} C^{2}$ TN, DECN, Instituto Superior Técnico, University of Lisbon, Estrada Nacional 10, P-2695-066 \\ Bobadela LRS, Portugal \\ ${ }^{3}$ Max-Planck Institute for Chemical Physics of Solids, Nöthnitzer Str. 40, D-01187 Dresden, Germany
}

Abstract. Quantum effects such as weak-antilocalisation (WAL) behavior and Shubnikov-de Haas ( $\mathrm{SdH}$ ) oscillations in the electrical transport properties of topological insulators, measured on nanostructured polycrystalline samples and single-crystals of a series of bismuth chalcogenide compounds $\left(\mathrm{Bi}_{2}\left(\mathrm{Se}_{x} \mathrm{Te}_{1-x}\right)_{3}\right.$, $0 \leq x \leq 1$ and $\mathrm{BiSbTe}_{3}$ ), are presented and discussed.

\section{Introduction}

Topological insulators (TIs) are celebrated novel materials with a peculiar band structure that features a gap for bulk states but may also host spin-polarised surface conducting states, topologically protected from scattering mechanisms that do not break time-inversion symmetry [1]. A representative family of such materials are bismuth chalcogenides, that have attracted a lot of interest also for their good properties as thermoelectric compounds [2].

The absence of back-scattering of the $2 \mathrm{D}$ electrons by non-magnetic impurities on TIs gives rise to a distinctive phenomenon known as weak anti-localisation (WAL) effect, a quantum correction to the classical magnetoresistance. It is characterised as a sharp cusp of the 2D magnetoconductance $\Delta \sigma_{x x}(B)$ at zero magnetic field. This quantum effect is well described in 2D systems by the Hikami-Larkin-Nagaoka (HLN) equation [3],

$$
\Delta \sigma_{x x}=\alpha \frac{e^{2}}{\pi h}\left[\Psi\left(\frac{\hbar c}{4 e l_{\phi}^{2} B}+\frac{1}{2}\right)-\ln \left(\frac{\hbar c}{4 e l_{\phi}^{2} B}\right)\right],
$$

where $\Psi$ is the digamma function, $l_{\phi}$ is the phase coherence length, and the prefactor $\alpha$ is a coefficient with a negative value close to $-1 / 2$ for the WAL, arising from the gapless surface states of TIs.

At high magnetic fields and low temperatures, $\mathrm{SdH}$ oscillations are often found in the electrical transport properties of TIs, as the Fermi level crosses the Landau levels of the 2D electron gas. Analysis of such oscillations provides invaluable information about the Fermi surface. The Lifshitz-Onsager equation relates the index $n$ of the $\mathrm{SdH}$ oscillations to the cross-section area of the Fermi surface perpendicular to the applied field,

$$
2 \pi(n+\gamma)=\pi k_{\mathrm{F}}^{2} \frac{\hbar}{e B}
$$

\footnotetext{
*Corresponding author: jap@ fis.uc.pt
} 
where $\pi k_{F}^{2}$ is the cross-sectional area of the Fermi surface perpendicular to $B$ with radius equivalent to the Fermi wave vector $k_{\mathrm{F}}$, and $\gamma=1 / 2-\beta-\delta$ is the phase of the SdH oscillations (in $2 \pi$ units) of the $\sigma_{x x}$ component of the electrical conductivity tensor, with $\beta$ the Berry phase and $\delta$ a phase shift correction determined by the dimensionality of the system. For conventional metals, $\beta=0$, and for Dirac systems it should be $\frac{1}{2}$. For a 3D electron system $\delta= \pm 1 / 8$, while for a 2D system $\delta=0$ [4]. Experimentally, the Berry phase can be obtained by plotting the index $n$ of the oscillations (position of the $\sigma_{x x}$ minima) versus $\frac{1}{B}$ [5]. The amplitude of the $\mathrm{SdH}$ oscillations decreases rapidly with temperature following the LifshitzKosevich law [6]. By measuring and analysing the temperature dependence, an important piece of information, the effective mass of the charge carriers can be obtained.

\section{Materials and Methods}

Nanostructured samples of $\mathrm{Bi}_{2}\left(\mathrm{Se}_{x} \mathrm{Te}_{1-x}\right)_{3}, 0 \leq x \leq 1$, were synthesised using a simple wet-chemical route previously reported [5,7]. Stoichiometric amounts of bismuth nitrate pentahydrated, sodium selenide and/or telluride and potassium hydroxide were dissolved in ethylene glycol. The solution was heated in a microwave digestion system with power, target temperature, heating and cooling times adjusted to achieve the best results. The reaction product is a precipitate that was centrifuged, washed with deionised water and ethanol, and later dried. Purity of the samples was checked by powder X-ray diffraction on a Bruker D8-Advance diffractometer (Fig. 1).

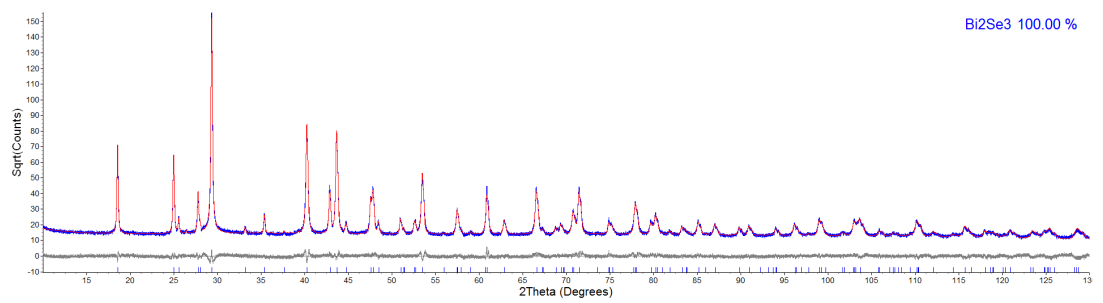

Figure 1. Powder X-Ray diffractogram of a nanostructured $\mathrm{Bi}_{2} \mathrm{Se}_{3}$ sample. No extraneous Bragg peaks of impurities were detected (the $y$ axis scale is depicted as the square-root of the intensity for better sensitivity to small peaks). The solid line passing through the measured points is the result of a Rietveld fit, the flat difference line between the measured and calculated intensities shown at the bottom further proves the sample purity.

Although this is a simple and fast synthetic method, fine adjustment of the heating conditions is necessary because extraneous phases are easily formed e.g. $\mathrm{Bi}$ and $\mathrm{Te}$ oxides, $\mathrm{Bi}_{2} \mathrm{Se}_{2} \mathrm{Te}$ and unreacted Te. Under optimised conditions, easier to attain for the end-member compositions, single-phase samples can be produced in the form of small nanoplates of hexagonal habit of micrometer size and nanometer width (Fig. 2).

Single crystals of $\mathrm{Bi}_{2} \mathrm{Te}_{3}$ and $\mathrm{BiSbTe}_{3}$ were grown by the Bridgman-Stockbarger method, starting from high purity elements $(6 \mathrm{~N})$ that were further purified by floating zone $(\mathrm{Bi}, \mathrm{Sb})$ and sublimation (Te) refinement before attempting crystal growth. As the electronic properties of these compounds are critically dependent not only on even small amounts of impurities but also on stoichiometry, slightly different deviations from the ideal composition were tried. Small pieces of the obtained crystals were tested to confirm the correct structure and composition using a single crystal X-Ray diffractometer (Bruker ApexII) and a SEM/EDS (Vega3 $\mathrm{SBH}$, Tescan) equipment. 


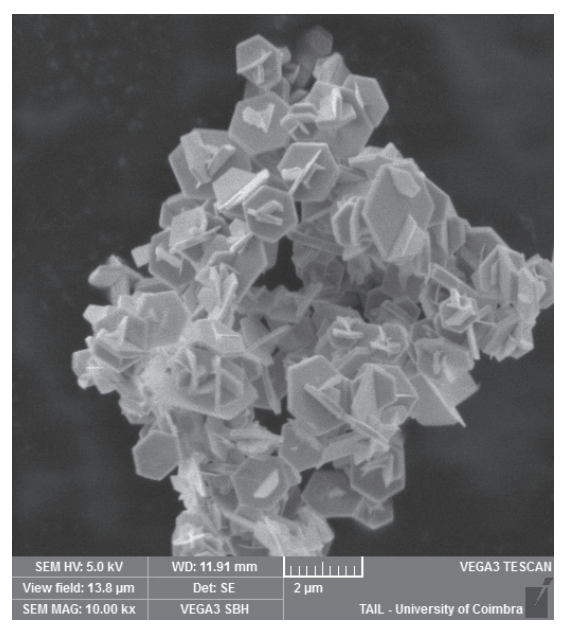

Figure 2. SEM image of the $\mathrm{Bi}_{2} \mathrm{Se}_{3}$ crystals produced using a wet-chemical method (see Sect. 2.)

\section{WAL in nanostructured Bismuth chalcogenides}

A strong WAL effect is a distinctive quantum phenomenon typically found in bismuth chalcogenide TIs. It has been observed in single-crystalline, polycrystalline and thin film samples. The electrical transport properties of our nanostructured samples were measured on small pellets formed by pressing the dried synthesised powder under a 6 MPa pressure. XRD data show that these pellets are strongly textured with the hexagonal $c$-axis perpendicular to the pellet surface. The electrical conductivity and Hall effect of such samples were measured as function of temperature $(1.8 \leq T \leq 300 \mathrm{~K})$ and magnetic field $(0 \leq B \leq 9 \mathrm{~T})$ on a Dynacool Quantum Design PPMS system. A particular attention needed to be taken to the electrical sample contacts, as the usual procedure of directly gluing thin gold wires into the sample with conducting silver paint resulted in a time-dependent resistivity arising from thermal diffusion of $\mathrm{Ag}$ atoms into the sample. Therefore, the electrical connections to the sample were made on $\mathrm{Au} / \mathrm{Pd}$ sputtered contacts on the sample surface that proved effective to strongly reduce the above mentioned diffusion effect. Fig. 3 shows the measured magnetoresistance at $1.8 \mathrm{~K}$ of the pure nanostructured $\mathrm{Bi}_{2} \mathrm{Se}_{3}$ sample discussed in Sect. 2.

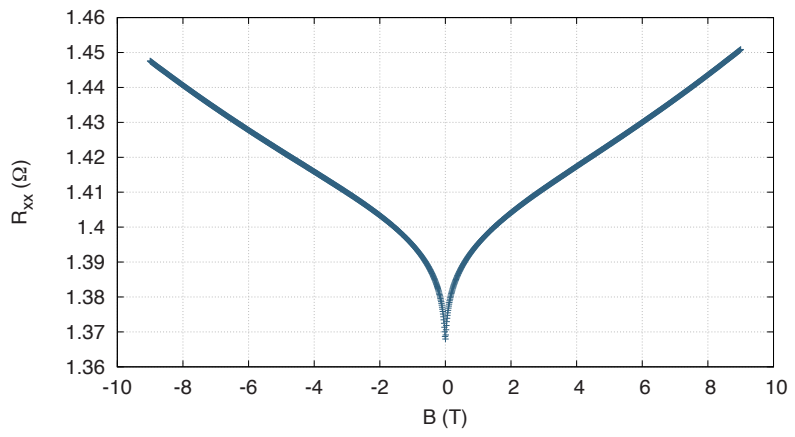

Figure 3. Magnetoresistance of a nanostructured $\mathrm{Bi}_{2} \mathrm{Se}_{3}$ sample measured at $1.8 \mathrm{~K}$, showing a strong weak-antilocalisation effect. 
Interestingly, WAL is a robust phenomenon that can still be easily observed in similar nanostructured samples even if they contain a significant amount of extraneous phases [5]. This surprising observation can be explained by the large coherence length of the electronic wave-functions at low temperatures. Its temperature dependence (Fig. 4) provides additional information regarding the dimensionality of the electron system: for $2 \mathrm{D}$ systems, and a dominant electron-electron dephasing mechanism, it is expected a power-law dependence $l_{\phi} \sim T^{-1 / 2}$, while for 3D systems $l_{\phi} \sim T^{-3 / 4}$ [9].
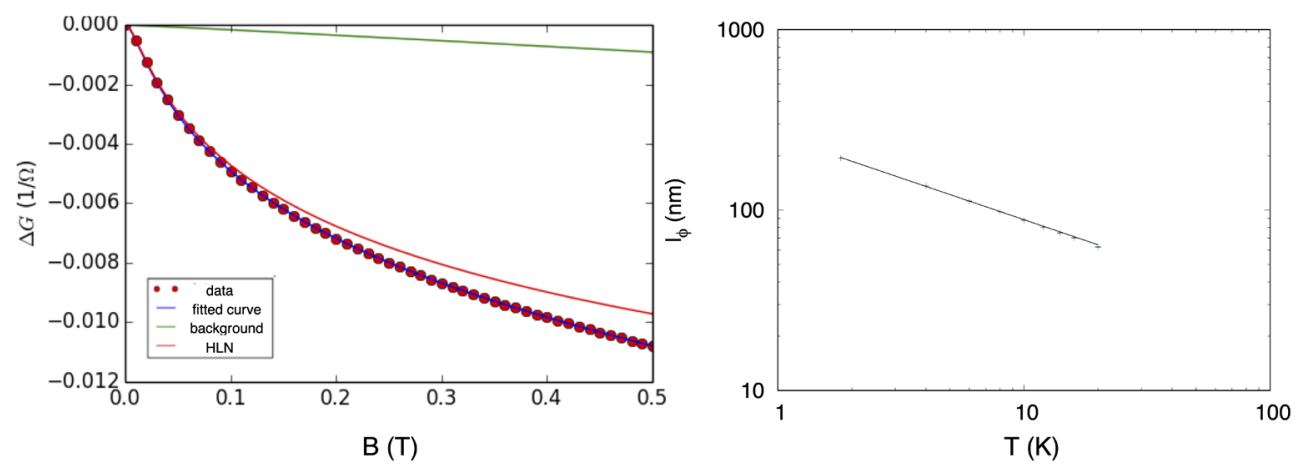

Figure 4. (left) Fit of the HLN model to the $\Delta G=1 / R_{x x}(B)-1 / R_{x x}(0)$ data of the nanostructured sample of $\mathrm{Bi}_{2} \mathrm{Se}_{3}$ measured at $1.8 \mathrm{~K}$. The fit yields a coherence length $l_{\phi}=193.96 \pm 0.17 \mathrm{~nm}$ at $1.8 \mathrm{~K}$. (right ) Power-law fit of coherence length dependence on temperature. The fit yields $l_{\phi} \propto T^{-0.463(5)}$, corroborating that the WAL effect originates from 2D electronic states. The fitted $\alpha=-3.67(1)$ value deviates significantly from the expected vale of -1.0 for two 2D conducting channels, at the top and bottom surfaces, but this parameter is prone to error due to the large uncertainty in estimating the geometrical factors when converting the measured bulk resistance into the 2D sheet conductance.

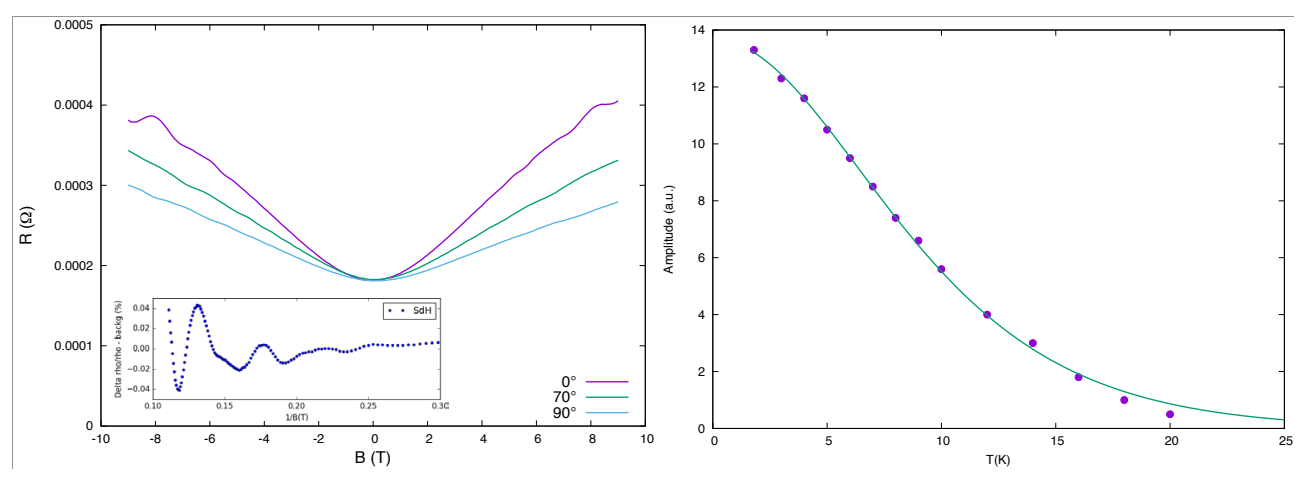

Figure 5. (left) Magnetoresistance measured on a single crystal of $\mathrm{BiSbTe}_{3}$ at $1.8 \mathrm{~K}$ for different angles between the applied magnetic field and the normal to the sample surface, showing SdH oscillations. (right) Fit of the Lifshitz-Kosevich model to the amplitude of the SdH oscillations, giving $m^{\star}=0.14 m_{0}$.

\section{Shubnikov-de Haas oscillations}

$\mathrm{SdH}$ oscillations in the electrical transport properties have been often observed in bismuth chalcogenide compounds. In contrast with the WAL behaviour, $\mathrm{SdH}$ oscillations are easier to 
observe when the samples depart from the typical semiconductor behaviour into the metallic regime, due the position of the Fermi level at a specific stoichiometry or to the presence of impurities that may act as electron donors or acceptors. We illustrate this phenomenon on data from a single crystalline $\mathrm{BiSbTe}_{3}$ sample grown as described in Sect. 2. The electrical resistivity shows a typical metallic behaviour with $\rho \sim 16 \mu \Omega \mathrm{m}$ and a $R R R \sim 15$. The measured thermoelectric power at room temperature is $S=130 \mu \mathrm{V} / \mathrm{K}$. The positive sign of the thermopower as well as the sign of the Hall coefficient show that the majority electric carriers are positively charged. i.e., are holes in the valence band.

The magnetoresistance measured as function of the applied field did not exhibit the lowfield cusp associated with WAL but at high fields $\mathrm{SdH}$ oscillations of the magnetoresistance are clearly observed. These oscillations are, as expected, stronger when the field is perpendicular to the sample surface and tend to vanish if it is parallel to the surface. The amplitude of the SdH oscillations was followed as function of temperature. The data shows a good fit to the Lifshitz-Kosevich law, as depicted in Fig. 5. The cyclotron effective mass of the dominant charge carriers obtained from such fit is $m^{\star}=0.14 m_{0}$, close to the calculated value of the hole mass in the valence band of $\mathrm{BiSbTe}_{3}[10]$.

\section{Conclusions}

We have observed distinctive quantum effects (WAL and SdH oscillations) in the electrical transport properties of TIs of the bismuth chalcogenides family. WAL is easier to find in those samples that are less conducting, and could be observed in nanostructured samples as well as in single-crystals. It is a particularly robust effect that was observed both in pure and impure samples. For the most conducting samples, the SdH effect was clearly observed and was analysed within the framework of the Lifshitz-Onsager model. The temperature dependence of the amplitude of the SdH oscillations follows the Lifshitz-Kosevich law [6]. Other quantum effects such as the Aharonov-Bohm effect have been recently reported [8] in $\mathrm{BiSbTe}_{3}$ adding to the interest of studying such TI compounds.

This work was partially supported by funds from FEDER (Programa Operacional Factores de Competitividade COMPETE) and from FCT under the projects UID/FIS/04564/2016 and PTDC/FISNAN/6099/2014. Access to instruments of the TAIL-UC facility funded under QREN-Mais Centro project ICT_2009_02_012_1890 is also gratefully acknowledged.

\section{References}

[1] Y. Hondo, J. Phys. Soc. Jpn. 82, 102001 (2013).

[2] D. Park, S. Park, K. Jeong, H-S. Jeong, J.Y. Song, M-H Cho, Scientific Reports 6, 19132 (2016).

[3] S. Hikami, A. I. Larkin and Y. Nagaoka, Prog. Theor. Phys. 63, 707 (1980).

[4] D. Kumar, A. Lakhani, Phys. Status Solidi RRL 9(11) 636-640 (2015).

[5] V.M.M. Pereira, M.S.C. Henriques, J.A. Paixão, Physica B 536 51-55 (2018).

[6] I.M. Lifshitz, A.M. Kosevich, Sov. Phys. JETP 2, 636 (1955).

[7] H. Xu, G. Chen, R. Jin, D. Chen, Y. Wang, J. Pei, Y. Zhang, C. Yan, Z. Qiu, CrystEngComm 16, 3965-3970 (2014).

[8] S. Huang, P. Wang, C. Lin, S. You, W. Lin, L. Lin, Y. Yan, S. Yu, M. Chou, Appl. Phys. Lett. 112, 203103 (2018).

[9] J. Liao, Y.B. Ou, H.W. Liu, K. He, X.C. Ma, Q.K. Xue, Y.Q. Li, Nat. Commun. 8, 16071 (2017).

[10] B.S. Farag, A.M.A. El Soud, H.A. Zayed, S.A. Gad, J. Ovonic Res. 6,267-275 (2010). 